\title{
Survival hydrodynamics
}

\section{S. Triantafyllou $†$}

Department of Mechanical Engineering, Massachusetts Institute of Technology, Cambridge, MA 02139, USA

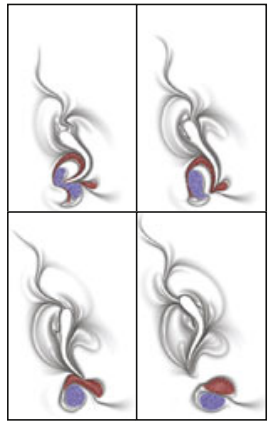

Fish escaping from predators exhibit amazing acceleration. The hydrodynamic mechanisms employed to power this acceleration are highlighted in the paper by Gazzola, van Rees \& Koumoutsakos (J. Fluid Mech., this issue, vol. 698, 2012, pp. 5-17), showing that the fish bends its entire body and caudal fin, in order to entrap and then accelerate as large a mass of water as possible. It is also shown that hydrodynamic optimization drives the fast-start kinematics.

Key words: swimming/flying, vortex dynamics

\section{Introduction}

The survival of fish depends on fast manoeuvres, whether they are escaping from predators, attacking prey, or jumping upstream in water falls. It is natural to expect them to employ every means to maximize their chances of success. Measured fish performance more than confirms this expectation; in fact, one can think of few instances when animal performance is more striking, as fish $30 \mathrm{~cm}$ long can reach accelerations exceeding 10 times gravity acceleration in a fraction of a second.

In a series of articles (reviewed in Domenici 2011), Webb explored the fascinating fast-starting performance of the trout, establishing the kinematics that lead to such accelerations: a first phase where the fish rapidly bends its body into a shape resembling either a letter $\mathrm{C}$ or a letter $\mathrm{S}$, and then a subsequent phase where the body is straightened in a travelling wave-like fashion, starting with the head and ending with the tail (Webb 1976). Subsequent work by Harper \& Blake (1991) and Domenici \& Blake (1997) provided data for other species, including another aggressive fish, the pike, whose performance is even more striking than the trout's, exceeding 10 times gravity acceleration.

A fast-start requires a carefully choreographed motion, because the fish must effect a very rapid motion of a suitable mass of water. The muscles used to bend the body and its fins must work at full capacity in a precisely timed manner. Although the available muscular power defines the limits of performance (Wakeling \& Johnston 1998), the use of suitable hydrodynamic mechanisms is essential to allow this escape to materialize.

Simple energetics dictate that the fish must accelerate as large a mass of water as possible: for a given required impulse, the energy conveyed to the fluid is proportional to the product of mass and velocity squared and, hence, is reduced as mass increases

$\dagger$ Email address for correspondence: mistetri@mit.edu 
and velocity decreases. The acceleration of fluid implies also the generation of vorticity, to be conveyed to the fluid as required by the kinematics of the accelerated flow; this vorticity must be generated at the body and fins of the fish.

In live fish there are several complicating factors. An escape manoeuvre is in response to a stimulus from a predator, hence it is part of a predator-prey interaction problem (Weihs \& Webb 1984). Also, the body form and use of fins increase the number of parameters that may affect performance; Weihs (1973) and Tytell \& Lauder (2008) have shown that fins can contribute significantly to the starting performance. The actuation and control of the musculature is equally important in achieving the required kinematics and to provide the required power. Finally, a C-shaped versus an S-shaped manoeuvre have different kinematic characteristics, although the levels of performance are similar.

Although these questions are very interesting for fish energetics, we would like first to tackle the basic question of whether the kinematics of the fast-start manoeuvres are optimal purely from the hydrodynamic point of view. Also, we investigate what are the basic hydrodynamic mechanisms, with the minimum number of shape and body parameters involved. The best way is to focus on a single type of manoeuvre of a simply shaped fish with only a caudal fin; and for which sufficient data are available. This is undertaken in the paper by Gazzola, van Rees \& Koumoutsakos (2012).

\section{Overview}

The paper by Gazzola et al. (2012), henceforth referred to as G-vR-K, explores and sheds new light on the hydrodynamics of zebrafish larvae C-starts. The authors test the hypothesis that fish maximize the escape distance, defined to be the motion of the fish's centre of mass at the end of the manoeuvre. Close similarity of the optimized manoeuvre with observed larva manoeuvres confirms the hypothesis.

The physical features and hydrodynamic performance of the zebrafish larvae are documented in a comprehensive paper by Mueller, van den Boogaart \& van Leeuwen (2008). The larva is not ideally streamlined (its $4.4 \mathrm{~mm}$ length provides a typical Reynolds number of 550, which is low) and its head bulges in a way that will cause separation (see figure 1 in $\mathrm{G}-\mathrm{vR}-\mathrm{K}$ ). This results in high Strouhal numbers of steady swimming (Mueller et al. 2008) and a wake consisting of multiple vortices per cycle. A C-start is dominated by inertia effects, however, so despite some flow separation evident in the simulations, one can safely extrapolate results to higher Reynolds numbers.

The optimization by $\mathrm{G}-\mathrm{vR}-\mathrm{K}$ allows the adjustment of parameters defining the magnitude and time evolution of the curvature of the fish; the problem is only constrained to qualitatively resemble the live fish C-start. The duration of the preparatory phase, as well as the duration of the propulsive phase are fixed.

The authors employ two-dimensional as well as three-dimensional simulations. Although the two-dimensional simulations may appear somewhat artificial, because the fish is modelled to be of infinite height, it is nonetheless very instructive, because flow visualizations of live fish at mid-height appear to be very close to twodimensional flow predictions (Wolfgang, Yue \& Triantafyllou 1999). The simulations provide confirmation of this result. In fact, the two-dimensional and three-dimensional optimized solutions are close (see figure 4 in $\mathrm{G}-\mathrm{vR}-\mathrm{K}$ ) to each other and the experimental results of Mueller et al. (2008), although there are also some basic differences. 
The first major contribution of $\mathrm{G}-\mathrm{vR}-\mathrm{K}$ is to establish that the kinematics of fast-start maximize escape distance. Since the time of manoeuvre is fixed, this is equivalent to maximizing mean speed or mean acceleration. As shown in figure $5(d)$ of $\mathrm{G}-\mathrm{vR}-\mathrm{K}$, distance is optimized, but not energetic efficiency. The observations on the mechanisms of fast-start are another major contribution of the paper, in particular through the use of 'tracer particles'. These are marked fluid particles, chosen to be contained in the vortical structures that form ultimately in the wake of the fish. By tracing back where these particles came from, we can determine how the various parts of the body and fins contribute to the formation of these patterns.

Using tracer particles becomes a great tool to connect the flow features during the manoeuvre (manoeuvring flow), when the flow is largely irrotational, except for the boundary layer of the fish; with the vortical flow obtained in the wake of the fish after the manoeuvre ends (wake flow). The vorticity shed in the wake flow can be traced back to the surface of the body and the caudal fin. What is of even greater interest is the connection of the vortical patterns in the wake flow, with the irrotational flow of the manoeuvring flow.

Figure 6 of G-vR-K summarizes a basic result of the study. In two-dimensional simulations (reproduced in the figure by the title) it is easy to establish that the flow 'captured' in the C-shape of the preparatory phase is either contained or surrounds the vortical patterns of the wake flow. The three-dimensional simulation results are qualitatively similar when plotted at the mid-plane of the fish. Figure 7 of G-vR-K adds important details of the three-dimensional flow, demonstrating the formation of two vortex rings, but more importantly the fact that the flow captured by the C-shaped bend spreads over a wide region. This is expected, because the irrotational-like part of the flow evolving in three dimensions contains a significant dipole-like structure at any particular planar cut perpendicular to the mid-line curve describing the shape of the fish (Zhu et al. 2002).

Documenting the transition from the largely potential flow features of the manoeuvring flow, to either two vortex pairs in flow restricted to two dimensions or two vortex rings in three-dimensional flow, is a major outcome of $\mathrm{G}-\mathrm{vR}-\mathrm{K}$. It is important to underline that the propulsive phase is in the form of a travelling wave; this is equivalent to rapidly pulling the body away, while retaining its shape (travelling wave), to release its energy and vorticity. In the study by Wibawa et al. (2012), a foil is pulled out while towed at an angle of attack, resulting in a nearinstantaneous formation of two very strong vortices entraining the added mass energy of the vanishing foil.

\section{Future}

G-vR-K have illustrated the mechanisms used by fish to achieve their spectacular fast-starting performance. The optimization method and tracer particles can be applied to investigate a number of other questions, especially if they are enhanced to allow detailed feature selection. For example, it would be very interesting to establish a hydrodynamic explanation of maximum acceleration and manoeuvre timing as function of fish size, and relate these quantities to the observed speeds of the travelling wave (Wakeling \& Johnston 1998). It would also be valuable to obtain the link between optimized fish morphology, fin shape and location, and kinematics, with hydrodynamics, to further illustrate the basic mechanisms of fast-start. The differences in the flow patterns of the S-starts are now relatively poorly understood. (Although such starts result in small turning angles for the fish, they lead to equally impressive 
accelerations.) Finally, the approach used in $\mathrm{G}-\mathrm{vR}-\mathrm{K}$ could be usefully applied to assessing the specific effect of the originally imparted kinetic energy during the $\mathrm{C}$-shape bending, as well as the precise process of its transfer to the fluid during the propulsive phase. This is important for biomimetics, as Conte et al. (2010) employed a mechanical fish that performed a fast-start starting from a bent position at rest, achieving qualitatively the same features but at reduced performance compared with the real fish.

\section{References}

Conte, J., Modarres-Sadeghi, Y., Watts, M. N., Hover, F. S. \& Triantafyllou, M. S. 2010 A fast starting mechanical fish that accelerates at $40 \mathrm{~m} \mathrm{~s}^{-2}$. Bioinsp. Biomim. 5, 1-9.

DoMENICI, P. 2011 Webb scales fast-start maneuvers. J. Expl Biol. 214, 875-877.

DOMENICI, P. \& BLAKE, R. 1997 The kinematics and performance of fish fast-start swimming. J. Expl Biol. 200, 1165-1178.

Gazzola, M., van Rees, W. M. \& Koumoutsakos, P. 2012 C-start: optimal start of larval fish. J. Fluid Mech. 698, 5-17.

HARPER, D. G. \& BlAKE, R. 1991 Prey capture and the fast-start performance of northern pike (Esox Lucius). J. Expl Biol. 155, 175-192.

Mueller, U. K., VAn den BoogaArt, J. G. M. \& VAn Leeuwen, J. L. 2008 Patterns of larval fish: undulatory swimming in the intermediate flow regime. J. Expl Biol. 211, 196-205.

Tytell, E. D. \& LAuder, G. V. 2008 Hydrodynamics of the escape response in bluegill sunfish, Lepomis macrochirus. J. Expl Biol. 211, 3359-3369.

Wakeling, J. M. \& Johnston, I. A. 1998 Muscle power output limits fast-start performance in fish. J. Expl Biol. 201, 1505-1526.

WEBB, P. 1976 The effect of size on the fast-start performance of rainbow trout (Salmo gairdneri) and a consideration of piscivorous predator-prey interaction. J. Expl Biol. 65, 157-177.

WEIHS, D. 1973 The mechanism of rapid starting of slender fish. Biorheology 10, 343-350.

WEIHS, D. \& WEBB, P. 1984 Optimal avoidance and evasion tactics in predator-prey interactions. J. Theor. Biol. 106, 189-206.

Wibawa, M. S., Steele, S. C., Dahl, J. M., Rival, D. E., Weymouth, G. D. \& Triantafyllou, M. S. 2012 Global vorticity shedding for a vanishing wing. J. Fluid Mech. 695, 112-134.

Wolfgang, M. J., Yue, D. K. P. \& Triantafyllou, M. S. 1999 Visualization of complex near-body transport processes in flexible-body propulsion. J. Flow Vis. 2, 143-151.

Zhu, Q., Wolfgang, M. J., Yue, D. K. P. \& Triantafyllou, M. S. 2002 Three-dimensional flow structures and vorticity control in fish-like swimming. J. Fluid Mech. 468, 1-28. 